\title{
Lecithin as a putative biodegradable blocker of SARS-CoV-2
}

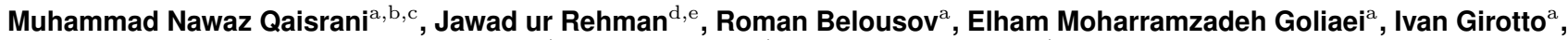 \\ Ricardo Franklin-Mergarejo ${ }^{\mathrm{a}}$, Oriol Güell ${ }^{\mathrm{f}, *}$, Ali Hassanali ${ }^{\mathrm{a}, *}$, and Édgar Roldán ${ }^{\mathrm{a}, *}$
}

a ICTP - The Abdus Salam International Centre for Theoretical Physics, Strada Costiera 11, 34151 Trieste, Italy; ${ }^{b}$ SISSA - International School for Advanced Studies, Via Bonomea 265, 34136 Trieste, Italy; ${ }^{\mathrm{c}}$ Institute of Physics, Johannes Gutenberg University Mainz, Staudingerweg 7, 55099 Mainz, Germany; ${ }^{\mathrm{d}}$ Dipartimento di Scienze Chimiche e Farmaceutiche, Università degli Studi di Trieste, Via Giorgieri 1, 34127 Trieste, Italy; ${ }^{\mathrm{e}}$ H.E. J. Research Institute of Chemistry, International Center for Chemical and Biological Sciences, University of Karachi, Karachi-75270, Pakistan; ${ }^{f}$ Comercial Douma S.L., Carrer de València 5, 08015 Barcelona, Spain

This manuscript was compiled on October 7, 2020

\begin{abstract}
Understanding the physical and chemical properties of viral infection at molecular scales is a major challenge of the scientific community in the fight against the Coronavirus (COVID-19) pandemic. We employ all-atoms molecular dynamics simulations to study the interaction between the receptor-binding domain of the SARS-CoV-2 spike protein and the surfactant lecithin in water. Our microsecond simulations reveal a preferential binding of lecithin to the receptorbinding motif (RBM) of SARS-CoV-2 with binding energies significantly larger than $k_{\mathrm{B}} T$. Furthermore, we find that these binding events are dominated by hydrophobic interactions involving the nonpolar tails which is simultaneously accompanied with a depletion of water near the RBM. Through an analysis of fluctuations in the RBD's radius of gyration, RBD-lecithin contact maps, and distributions of water molecules, our simulations reveal the possible role of lecithin as a flexible blocker of SARS-CoV-2.
\end{abstract}

SARS-CoV-2 | Coronavirus | COVID-19 | Spike protein | Lecithin | Molecular dynamics

$\mathbf{O}$ ver the last century, humanity has been threatened by several deadly viruses including Spanish flu, SARS Coronavirus (SARS-CoV), Influenza A (H1N1, H5N1), Ebola, Middle East Respiratory Syndrome (MERS-CoV), Zika, and recently Coronavirus disease 2019 (COVID-19 or SARS-CoV-2) (1-3). To date, the total number of COVID-19 positive cases are in the order of millions according to the World Health Organization (4). Due to its rapid transmission and high rate of mortality, efforts from different disciplines are being put together in the global endeavour of tackling the Coronavirus pandemic.

The molecular structure of SARS-CoV-2 is formed by a lipid membrane, nucleocapsid proteins, and spike proteins, which together shield the RNA genome of the virus. It has been shown that spike proteins play a key role in the virus fusion and entry (5), hence they have become one of the key targets for drug design and vaccine development (6-9). SARS-CoV-2's spike protein is formed by two subunits, denoted by $\mathrm{S} 1$ and $\mathrm{S} 2$. The S1 subunit binds through its receptor binding motif (RBM) to the ACE2 (Angiotensin-converting enzyme 2) on the cell membrane surface of the lung. On the other hand, the S2 subunit mediates the fusion of the virus with the human host cells (10). Recent work has shown that SARS-CoV-2 spike protein binds to ACE2 human receptor with approximately $15 \mathrm{nM}$ affinity which is higher than the affinity of SARS-CoV $\mathrm{S}$ to ACE2 (11) and it leads to increased virulence of COVID19 (12-14). The protein and ACE2 can interact via hydrogen bonding, hydrophobic and electrostatic interactions (15-17). There have been numerous attempts to design or repurpose drugs that would inhibit the binding of the spike protein to
ACE2 (18-24). However, the underlying physical principles that drive these interactions are yet poorly understood. To explore novel and potent drugs, a fundamental understanding of the interplay between molecular forces involving the spike protein, potential inhibitors and of course the surrounding aqueous medium, is critical.

Numerous molecular dynamics studies are nowadays aiming to discover potential drugs that could serve as inhibitors or blockers for SARS-CoV-2 (25-28). It is well established that the binding affinity for drug-candidates is modulated by a subtle balance of interactions involving hydrogen bonding, electrostatics and hydrophobic effects. In this regard, it is interesting to wonder whether "soapy" molecules which have a hydrophobic and hydrophilic domains, could serve as flexible blockers in this problem. This is not completely unprecedented since phospholipids have been employed as carriers for drug molecules in the treatment of several viral diseases (29-31) but also other diseases such as high cholesterol (32). However, the possibility of using amphiphilic molecules to block the RBM binding domain of SARS-Cov-2 has not been previously explored.

Herein, we present proof-of-concept simulations that demonstrate that the binding sites of SARS-CoV-2 interacting with ACE2 in water can be blocked by ampiphillic biomolecules. Specifically we probe, with microsecond timescale atomistic molecular dynamics simulations, the efficacy in blocking SARS-

\section{Significance Statement}

The current COVID-19 pandemic has triggered a global scientific effort in the search for compounds that may inhibit the SARS-CoV-2 virus. Here, we use microsecond timescale molecular dynamics simulations to show that lecithin POPC, which can form liposomes and biological membranes, could play an important role in blocking the spike protein receptorbinding domain of SARS-CoV-2. Hydrophobic interactions, as well as water reorganization near the binding region, appear to play a critical role in the mechanism by which lecithin binds. Our results open up the possibility of using biodegradable surfactant molecules in the development of antiviral therapies.

$\mathrm{MNQ}$ and $\mathrm{EG}$ performed simulations. $\mathrm{MNQ}, \mathrm{JR}, \mathrm{RB}$, and RF analyzed data. $\mathrm{MNQ}$, JR, and $\mathrm{OG}$ discussed physico-chemistry aspects. IG provided computing support. OG, AH, and ER proposed and established the project, and $\mathrm{AH}$ and ER directed it. All authors discussed and wrote the manuscript.

The authors declare no competing interests.

"To whom correspondence should be addressed. E-mails: oriol@comercialdouma.com (OG), ahassana@ictp.it (AH), edgar@ictp.it (ER). 
CoV-2 receptor binding domain with an amphiphilic molecule, phosphatidylcholine (POPC). POPC is biodegradable, it can be extracted from both vegetable oils (e.g. soybean) and animal tissues (e.g. eggs) (33), and forms the phospholipid constituent of a commercially well-known product called lecithin. We show that lecithin molecule appears to form strong and stable aggregates driven by hydrophobic interactions and dewetting, in close proximity to the RBM and may thus hinder interactions with the ACE2 receptor.

The role of water and hydrophobicity in viral inhibition is not unprecedented. Specifically, recent works have shown that water plays an important role in the inhibition of SARS-CoV-2 via hydrophobic interactions by CB6 antibody and EK1C4 inhibitor $(34,35)$. Interestingly, the role of hydrophobicity and hydrogen bond networks have also been implicated in the interactions of both Zika and HIV viral membranes constituted by POPC bilayers $(36,37)$. An understanding of the the physico-chemical interactions of the spike protein with surfactants is also important because the depletion of pulmonary surfactant in the lungs is also a critical factor in respiratory related problems (38).

In the following we study, using molecular dynamics simulations, the interaction between SARS-CoV-2 receptor binding domain (RBD) and a phospholipid, amphiphilic molecule, at different concentrations in water. More precisely, we focus on POPC, which we will refer to throughout the paper as lecithin. We show that this molecule binds preferentially the receptor binding motif (RBM) of the spike protein driven by the formation of non-polar contacts. Furthermore, lecithin significantly alters the secondary structure of the spike protein which is strongly modulated by the concentration of the phospholipid. Notably, all these processes are accompanied by a significant change in the water local density at the vicinity of the RBM interface. These results open up the possibility for using surfactant molecules in drug design.

\section{System Setup}

In this section we introduce and discuss physicochemical properties of the system in which we focus our study, the SARS$\mathrm{CoV}-2$ receptor binding domain (RBD) and lecithin in water.

A. SARS-CoV-2 receptor binding domain. Figure $1 \mathrm{~A}$ illustrates the crystal structure of SARS-CoV2 RBD monomer (multi-colour) bound to the human ACE2 receptor (green)(27, 39). A zoomed-in view of the RBD crystal structure is displayed in Fig. 1B with the RBM highlighted in red. The residue sequence and its cryo-EM structure of spike glycoprotein's RBD were adopted from Refs. $(27,39)$. In order to dissect, and better interrogate the protein-lecithin interactions we divided the RBD into non-overlapping zones. Fig. 1B shows the five different regions in different colors in the protein structure labelled as Zone 1 (orange), Zone 2 (cyan), Zone 3 (yellow), the receptor-binding motif RBM (red) and Zone 4 (purple). The RBM is the largest domain and carries the residue sequence from Asn439 to Gln505 which has been shown to interact with human ACE2 receptor.

B. Lecithin. While it is well known that surfactant-protein interactions play an important role in biological contexts, the underlying physical forces that drive these processes remain poorly understood (40). Here, we focus our efforts

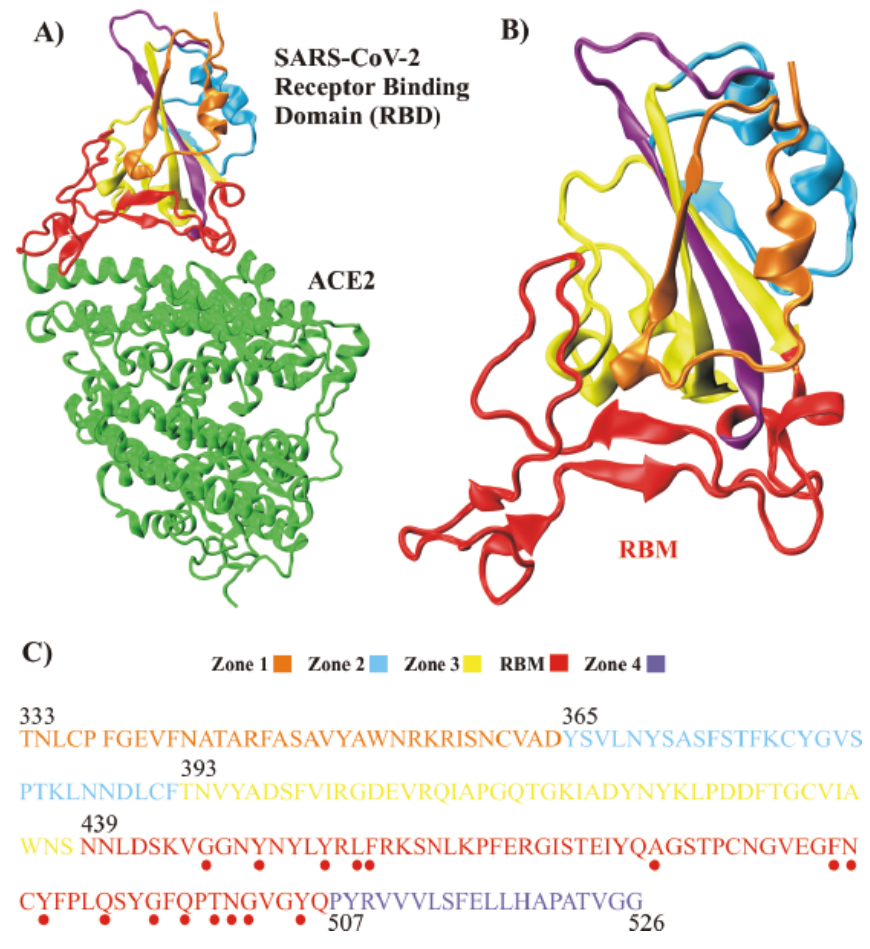

Fig. 1. Structures of SARS-CoV-2 spike protein receptor binding domain (RBD) and Angiotensin-Converting Enzyme 2 (ACE2). A) Illustration of the X-ray crystal structure of SARS-CoV2 spike protein receptor binding domain (RBD, multicolor) and human ACE2 receptor (green) $(27,39)$. B) Zoomed view of SARS-CoV-2 RBD, where different colours indicate different zones selected to interrogate the RBD-lecithin interactions at different solvent exposures. The red highlighted part is the receptor-binding motif (RBM) of the protein. C) Amino acid sequence corresponding to the crystal structure of SARS-CoV-2 RBD with the different zones highlighted in different colors. The red dots below few residues indicate those amino acids involved in the interaction with the human ACE2 receptor.

on the phospholipid POPC (1-palmitoyl-2-oleoyl-sn-glycero-3phosphocholine $\mathrm{C}_{42} \mathrm{H}_{82} \mathrm{NO}_{8} \mathrm{P}$ ), which we will refer throughout the paper as lecithin, a biodegradable, essential phospholipid for the human body. Lecithins are ubiquitous in mammalians organs, they help to build the largest choline reservoir and they are found in the bile (41), and more importantly in the alveolar surface in the lung $(42,43)$. Lecithin's chemical structure is shown in Fig. 2A. It is a surfactant consisting of a glycerol backbone sterified in positions 1 and 2 with a palmitic and an oleic acid, both constituting the hydrophobic non-polar tail able to interact with non-polar residues. The position 3 of the backbone is linked to a phosphate group which is bonded to a choline group, forming the hydrophilic polar head of lecithin.

Lecithin is amphiphilic and therefore has potential to interact with the spike protein using a combination of both polar and non-polar interactions. In fact, the hydrophiliclipophylic balance (HLB) of lecithin ranges from values to $4 \pm 1$ to $9.7 \pm 1$ (44). Thus, it lies in the range of w/o (water in oil) emulsifying agents and also of wetting spreading agents. This is a crucial property that makes lecithin POPC an ideal candidate to act as a molecule targeting SARS-CoV-2, since one will need both polar and non-polar parts, the former related to the polar amino acids of proteins, whereas the latter involves the protein hydrophobic regions. Moreover, at large concentrations lecithin can aggregate into micelles (32). As we will see later, the aggregation of lecithin in close proximity 
A)

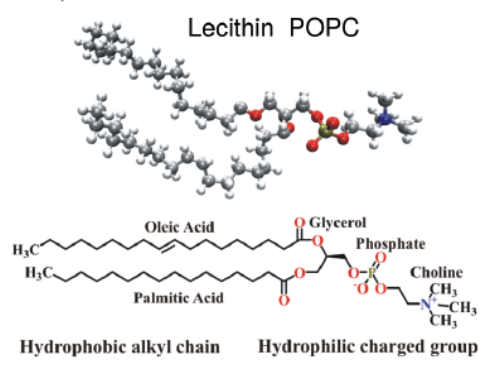

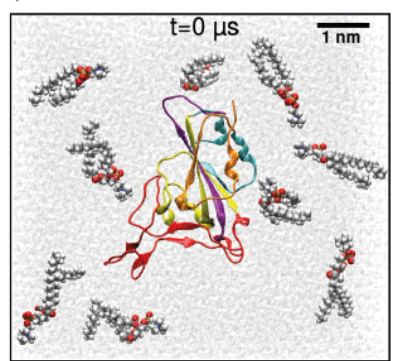

C)

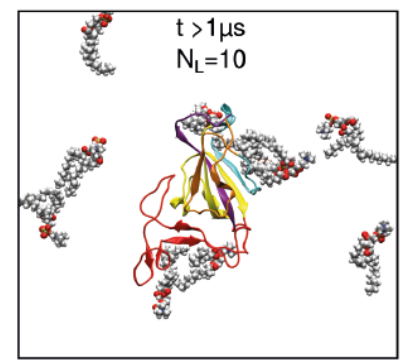

D)

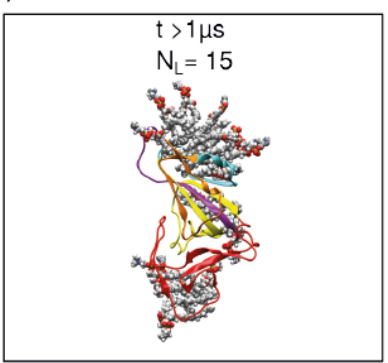

Fig. 2. Lecithin: chemical structure and interaction dynamics with SARS-CoV-2 RBD. A) Molecular (top) and chemical (bottom) structure of a lecithin POPC (1-palmitoyl-2oleoyl-sn-glycero-3-phosphocholine) molecule. The bottom figure highlights its polar (hydrophilic) and non-polar (hydrophobic) chemical groups. B) Snapshot of one initial condition of the simulation setup at, showing the receptor binding domain of SARS-CoV-2 spike protein in the lecithin solution, with water molecules depicted as transparent gray spheres. C-D) Snapshots taken at times larger than $1 \mu s$ for two different values of the lecithin concentrations corresponding to $N_{L}=10$ (C) and $N_{L}=15$ (D) number of lecithin molecules in the simulation box. For the sake of better visualization, we do not include the water molecules in panels $C$ and $D$.

to the spike protein might serve as a blocking mechanism for ACE2 binding with SARS-CoV-2.

\section{Results}

In order to build our intuition on the nature of the interactions between lecithin and the spike protein, we begin by inspecting representative snapshots of our molecular dynamics simulations (see Supplementary Movies 1, 2, and 3 for graphical representations of simulations done with lecithin concentrations $N_{L}=5, N_{L}=10$, and $N_{L}=15$ respectively). Figure $2 \mathrm{~B}$ shows the initial condition in a zoomed-in snapshot of the simulation box for $N_{L}=10$ lecithin molecules. Over time, lecithin molecules - initially at random positions in the simulation box - diffuse into the hydration shell of the protein and appear to have an affinity for certain regions of the spike protein. More specifically, we observe that lecithin molecules adhere to the RBM zone as well as Zone 2. Figures 2C-D are representative snapshots taken after $1 \mu \mathrm{s}$ simulation time for lecithin concentrations corresponding to $N_{L}=10$ and $N_{L}=15$ illustrating this phenomenon.

There are several interesting features that one can observe in these binding events of involving lecithin. Firstly, lecithin molecules stick around the RBM and Zone 2 either as single molecules (Fig. 2C) or in the form of clusters (Fig. 2D). Furthermore, the simulations also reveal that lecithin docks to the viral protein mostly involving the hydrophobic non-polar tail. Overall, Figure 2D shows the aggregation of lecithin into two clusters, one near the RBM and another in close proximity to Zone 2 (cf. Fig. 1B). Note that Zone 2 is exposed to the solvent in our simulations, but is bound to the core of the spike protein in the virus, hence it may be accessible to lecithin when the spike-protein trimer opens due to fluctuations or interactions with other proteins.

In the following, we provide a quantitative analysis of our simulations, by first looking in Sec. 2A at the global structural behavior of the SARS-CoV-2 RBD monitoring its radius of gyration. To investigate the protein-lecithin interactions at the atomic level, we present in Sec. 2B a detailed contact map analysis disentangling the interactions involving the polar and non-polar groups of the lecithin molecules with different SARSCoV-2 RBD residues. We then provide in Sec. $2 \mathrm{C}$ further insights about the energetics of the binding events reporting estimates of the binding energetics of lecithin molecules with
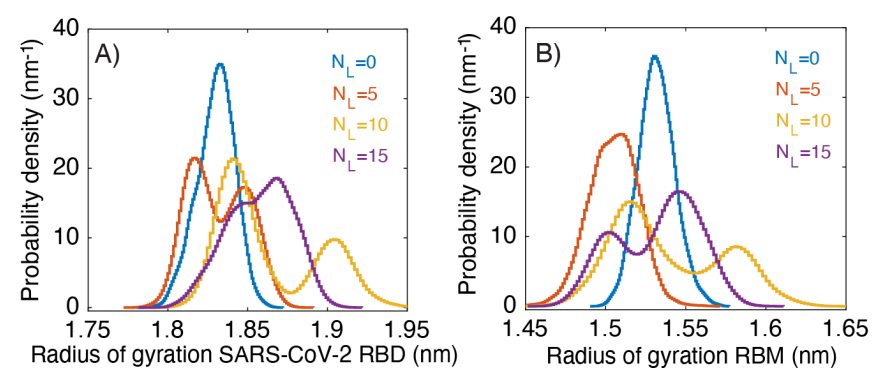

Fig. 3. Distribution of radius of gyration of SARS-CoV-2 spike protein receptor binding domain RBD (A) and of its receptor binding motif RBM (B) for different concentrations of lecithin (see legend, where $N_{\mathrm{L}}$ denotes the number of lecithin molecules). The data is obtained from a single $1 \mu \mathrm{s}$ molecular-dynamics simulation for each lecithin concentration value.

RBD. Next we show in Sec. 2D that the interaction of the hydrophobic parts of lecithin with the spike protein, reveals the crucial role of water during the interaction of the spike protein with its aqueous environment.

A. Radius of gyration. To monitor the change in the structure of SARS-CoV-2 RBD resulting from its interaction with lecithin, we measure the radius of gyration (RG) of the RBD as a function of time. In particular, we evaluate $\mathrm{RG}(t)=\left(\sum_{i} m_{i}\left\|\mathbf{r}_{i}(t)\right\|^{2} / \sum_{i} m_{i}\right)^{1 / 2}$, where $m_{i}$ and $\mathbf{r}_{i}(t)$ is the mass and position of each $i$-th atom respectively, with respect to the center of mass of the molecule to which it belongs to. We consider here two different RGs of interest: (i) RG of all the heavy atoms in the SARS-CoV-2 RBD; and (ii) RG of all the heavy atoms in the RBM. The distribution of the RGs (Figs. 3A-B) show that lecithin significantly changes the structure of the RBD and in particular, of its RBM, and that this effect is highly sensitive to the concentration of lecithin in water. These results show that the radius of gyration of both the RBM and the full RBD tend to increase when increasing the concentration of lecithin molecules.

B. Atlas of molecular interactions between lecithin and SARS-CoV-2 RBD. In this section, we investigate the interatomic contacts that form between lecithin and spike protein. To this aim, we plot in Fig. 4 contact maps between all the 

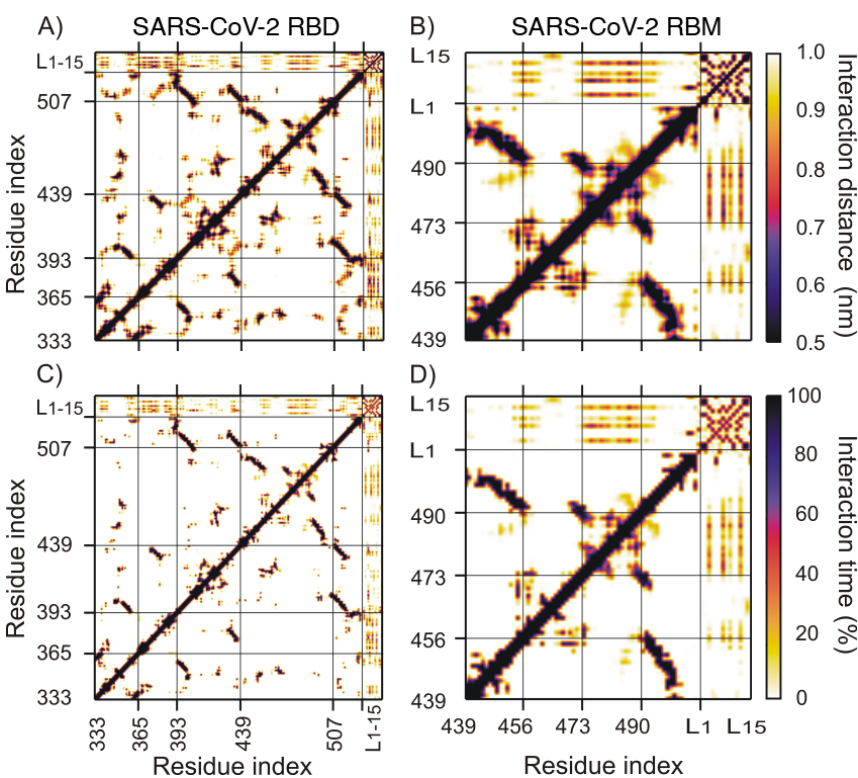

D)

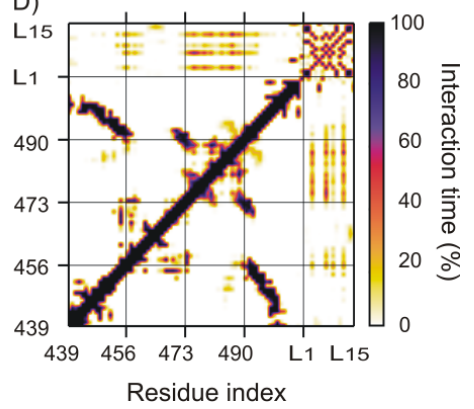

Fig. 4. Contact maps for interactions between different zones of SARS-CoV-2 receptor binding domain (RBD) and lecithin molecules (L). A) Contact map of distances for the whole RBD. B) Zoomed-in view of (A) for the receptor-binding motif (RBM). C) Contact map of interaction times for the whole RBD, at distances below $1 \mathrm{~nm}$. D) Zoomed-in view of $(C)$ for the receptor-binding motif (RBM). In (C-D) the time is expressed in \% relative to the total simulation time.

heavy atoms* in the protein residues of SARS-CoV-2 RBD and lecithin molecules for the lecithin concentration value $N_{L}=15$. We focus this analysis on two quantities, namely the average interatomic distance (Fig. 4A-B) and the average interaction time (Fig. 4C-D) between lecithin and the different viral protein zones (see Fig. 1). Figs. $4 \mathrm{~A}$ and C, show the interaction distances and times that lecithin forms with the entire RBD. Consistent with Fig. 2, we observe that there are some hot-spot regions where lecithin prefers to bind, in particular, Zone2 and RBM. Within these regions, the interatomic distances between lecithin and the viral protein are less than one nanometer. Since the RBM zone is the one that interacts with ACE2 we focus on examining its contacts with lecithin in Fig. 4B. Specifically, lecithin appears to form close contacts with amino acids 473-490, which contain ACE2-interacting residues (cf. Fig. 1C).

Figures $4 \mathrm{C}, \mathrm{D}$ show contact maps of the interaction times associated with lecithin and the RBD. In order to quantify interaction times, we count the time elapsed within a threshold distance of $1 \mathrm{~nm}$ corresponding to the typical range of vander-Waals forces. We find that the average interaction time between lecithin and the RBD is on the order of hundreds of nanoseconds. As expected, the core of the protein (Zone 3) is characterized by the weakest binding. We observe similar trends in our simulations at lower concentrations of lecithin, i.e for $N_{L}=5$ and $N_{L}=10$ lecithin molecules (see the Supporting Information).

To gain further insights into the physical driving force between the molecular interactions, we investigate the average contact maps of polar and non polar groups of lecithin molecules with the residues of the RBM. We evaluate the average interatomic distance between the RBM atoms and

* All our contact maps are evaluated taking into account the positions of all except the hydrogen atoms, i.e. only the heavy atoms.
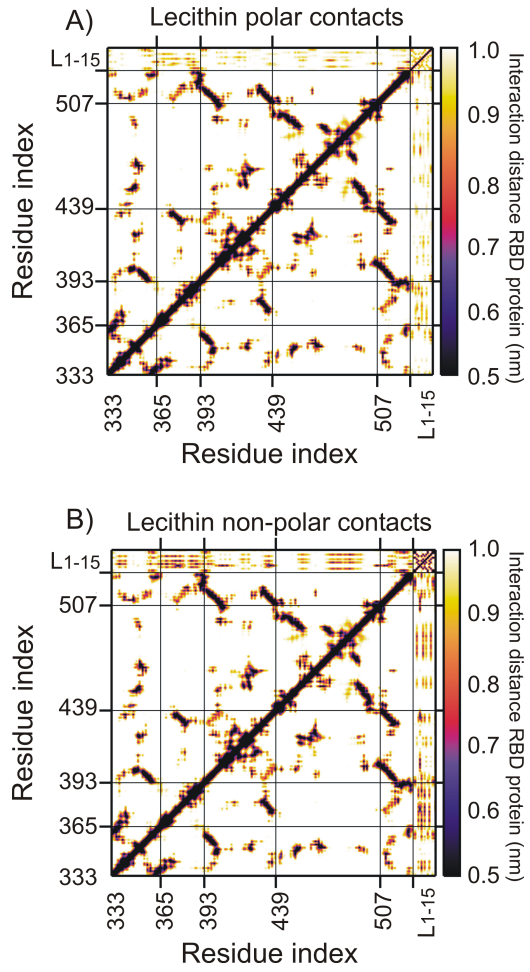

Fig. 5. Average contact maps between different zones of SARS-CoV-2 receptor binding domain (RBD) and lecithin polar (A) and non polar (B) groups, see text for more details.

two different parts of lecithin molecules: (i) the hydrophilic polar group (see Fig. 5A); and (ii) the hydrophobic nonpolar chain (see Fig. 5B). Notably, the interatomic distance between all the subdomains of the RBM and the hydrophobic tail of lecithin molecules is significantly smaller than the distance to the hydrophilic head. Therefore the lecithin-RBM contacts are mainly dominated by the hydrophobic interactions and in fact, this is enhanced under higher concentrations of lecithin.

C. Lecithin-RBM binding energetics. Our preceding results from the contact maps clearly point to the fact that lecithin can form favorable hydrophobic interactions with the RBM domain. It is beyond the scope of the current work to determine the binding free energies using the full atomistic simulations. Instead, we turned to using estimates given by the molecular mechanics Poisson-Boltzmann surface area (MM-PBSA) as implemented in Gromacs (45) to investigate the binding energy of lecithin to the protein over the course of the microsecond simulations we performed. This approach has been successfully used in numerous other biophysical applications (46-50).

Figure 6 shows the evolution of the binding energy estimate per lecithin molecule over the course of our microsecond simulations in solution with $N_{L}=15$ lecithin molecules. The three curves show that the change in interaction energy computed from the MM-PBSA at zero salt concentration, covers the range $-20 k_{\mathrm{B}} T$ to $-5 k_{\mathrm{B}} T$ per lecithin molecule. We have also found that the binding energy is enhanced to the range $-25 k_{\mathrm{B}} T$ to $-15 k_{\mathrm{B}} T$ when including an ionic concentration of $0.15 \mathrm{M}$ in our estimates (see Supporting Information). This result is also consistent with the fact that we observe few detachment events of lecithin molecules from the RBD in these 


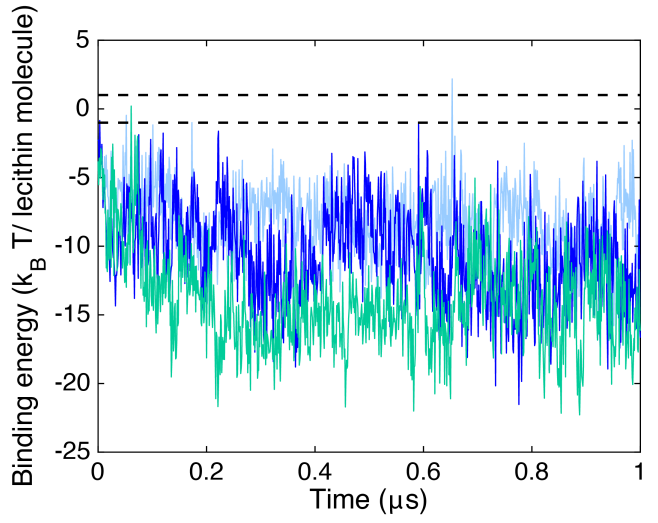

Fig. 6. Estimate of the binding energy (in units of $k_{\mathrm{B}} T$ per lecithin molecule) between lecithin and lecithin SARS-CoV-2 RBD as a function of time, obtained by applying the MM/PBSA method to our molecular dynamics in solution with $N_{L}=15$ lecithin molecules. The different lines (blue, cyan, teal) are obtained for three different initial conditions and the horizontal dashed lines are set to $\pm k_{\mathrm{B}} T$. In our MM/PBSA estimates we set the ionic concentration to $0 \mathrm{M}$.

simulations that are triggered by $\sim k_{\mathrm{B}} T$ energy fluctuations. Interestingly, our estimates are comparable to the binding affinity of different drug molecules with the SARS-CoV-2 $\mathrm{RBD}$ trimer ranging from $-5 \mathrm{kcal} / \mathrm{mol}$ to $-12 \mathrm{kcal} / \mathrm{mol}(51)$. A recent thorough study using molecular docking identified approximately 100 natural product inhibitors with binding energies of about $-9 \mathrm{kcal} / \mathrm{mol}$ to the TMPRSS2 protease (52). In conclusion, the binding energetics of lecithin to the RBM domain observed in our simulations suggests that phospholipids play an important role in the interactions involving the RBM domain under cellular conditions.

D. Dewetting of the receptor-binding motif. It is well known that water plays an instrumental role in tuning the structural and dynamical properties of biological systems (53). In the context of our work, numerous studies emphasized depletion of the hydration shell as an important factor facilitating the facilitating the hydrophobic interactions between proteins $(54,55)$. The preceding analysis shows that hydrophobic interactions between the non-polar groups of lecithin form close contacts with the RBM.

We now explore the reorganization of the solvent environment during the RBM-lecithin binding in water. We calculated the radial distribution of water molecules $g(r)$ for sixteen residues highlighted as "RBM hotspots" with red circles in Fig. 1C. These hotspots have been implicated in the docking mechanism of SARS-CoV-2 and ACE2 (56). Figure 7 shows the radial distribution functions of water near two RBM hotspots - hydrophobic residues, alanine Ala475 and phenylalanine Phe486. We observe a significant depletion in the water density over a length scale of $1 \mathrm{~nm}$ near both these amino acids in the presence of lecithin (solid lines in Fig. 7) with respect to their value in pure water (dashed lines in Fig. 7) for $N_{L}=15$. Figure 8 shows the hydration shell around Phe 486 in the absence and in the presence of a lecithin cluster. Interestingly, there is a rather wide spectrum of changes in the water density around the RBM hotspots which in part reflects their respective solvent exposure.

To gain further insights, we determined the hydration shell outer radius $r_{\mathrm{o}}$, defined as the position of the Gibbs dividing interface, for the RBM hotspots with and without lecithin

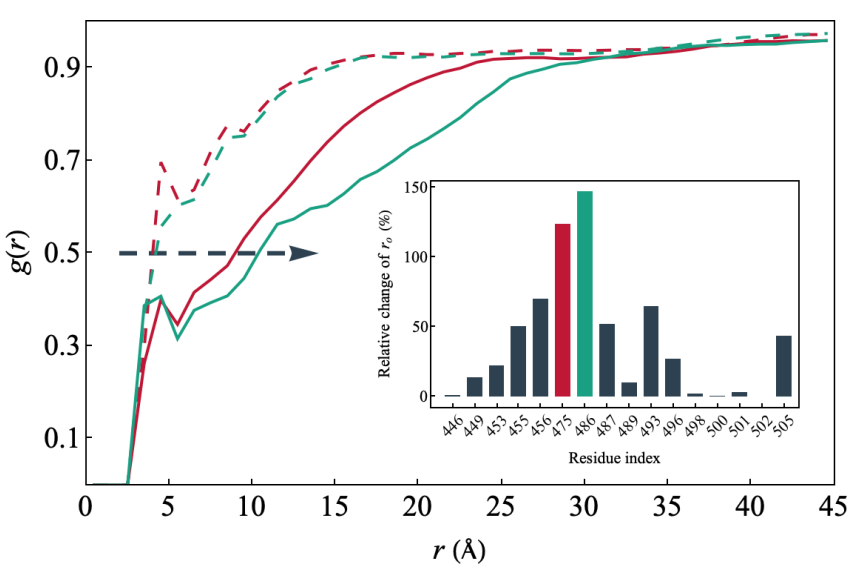

Fig. 7. Radial distribution function $g(r)$ of water molecules plotted as a function of distance $r$ from the 16 amino acids of the RBM domain which are involved in binding with ACE2 (highlighted as red dots in Fig. 1C). The analysis is done for a single $1.2 \mu \mathrm{s}$ long trajectory of system with $N_{L}=15$ (solid lines) and $N_{L}=0$ (dashed lines) lecithin in water solution respectively. The horizontal line illustrates the increase of the hydration shell's outer radius $r_{\mathrm{O}}$, which is delimited by the position of the Gibbs dividing interface $g\left(r_{\mathrm{o}}\right)=0.5$. We reproduce $g(r)$ only for the amino acids Ala475 and Phe486, near which the hydration shell is depleted the most due to the hydrophobic interactions with the lecithin molecules. The inset reports the percentage change of $r_{\mathrm{o}}$ for all the sixteen residues that we examined. Ala475 and Phe486 are singled out by the same colors as in the main plot. Note that as reference positions of the $\mathrm{H}_{2} \mathrm{O}$ groups and amino acids we chose the oxygen and the alpha carbon atoms, respectively.

in solution. Notably, $r_{\mathrm{o}}$ increases upon addition of lecithin for all these residues. This effect is most pronounced at large concentrations of lecithin (see Supporting Information). The inset of Fig. 7 shows the relative change of the sixteen amino acids $r_{\mathrm{o}}$ in the RBM-ACE2 junction. All sixteen undergo a depletion of water density to different extents, with residues Ala475 and Phe486 displaying a striking increase of more than $100 \%$.

\section{Discussion}

In this work we have studied with molecular dynamics simulations the interaction between SARS-CoV-2 receptor binding domain (RBD) and lecithin molecules in water. Our main results are summarized as follows: (i) lecithin induces a conformational change in the RBD and its receptor binding motif which is revealed by an increase of the radius of gyration with the lecithin concentration; (ii) lecithin molecules bind mostly by docking their hydrophobic tails into the receptorbinding motif (RBM) domain located within the RBD. This hydrophobic interaction occurs at distances that are smaller than $1 \mathrm{~nm}$ and with significant binding energies ranging from 10 to $20 k_{\mathrm{B}} T$ per lecithin molecule. (iii) The lecithin-RBD hydrophobic binding is accompanied by a dewetting of water molecules in the receptor binding motif. Taken all together, these results suggest the possible role of soapy-like molecules as potential biodegradable blockers of the interaction between SARS-CoV-2 RBD and ACE2 receptor.

The results obtained in this manuscript provide some fundamental basis of the possible action of lecithin, and the key role of hydrophobic interactions and water in the interactions involving the spike protein. On the one hand, liposomes built from lecithin provide a way to encapsulate possible drugs or mixtures of compounds that may inhibit the infection (57). 

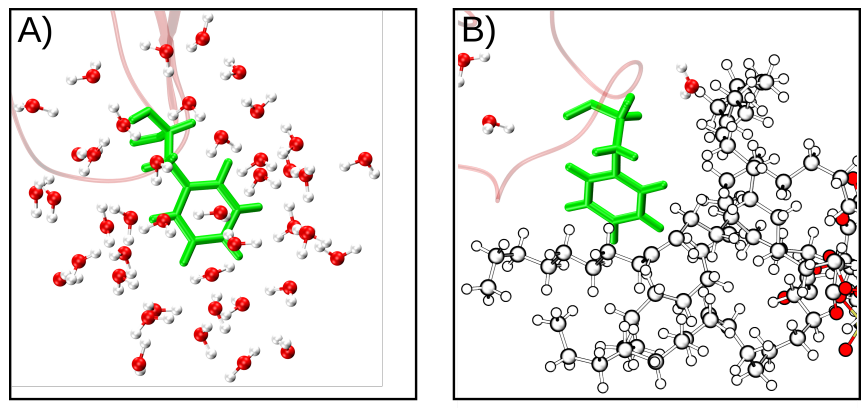

Fig. 8. Zoomed-in Snapshot taken from the simulation of the system where protein is in pure water (A) and when the protein is in lecithin solution (B). The hydration water within a radial distance of $5 \AA$ from the Phe 486 residue (in green) which belongs to the RBM (transparent red coils) is shown in both cases. In the vicinity of lecithin cluster (shown by glassy balls and sticks) when it formed near the RBM residues over hundreds of nanoseconds simulation time, the hydration shell is significantly depleted due to the hydrophobic interaction of lecithin molecules hence dewetting of the protein residues.

At the same time, lecithin itself appears to play an active role and maybe used in combination with other therapies. Note that this idea is not unwarranted as phospholipids have also employed tried to treat coronavirus (29). In fact, it has been successfully applied in therapies to reduce the amount of cholesterol (32), and in other antiviral therapies (30,31). Other approaches could be used as prevention techniques involving lecithin and other excipients and drugs that can help to reduce and to eventually eliminate SARS-CoV-2. Such techniques may be developed in easy-to-use and harmless ways such as nasal sprays, pills or emulsions. In a time where a microscopic understanding of the COVID-19 global pandemic remains unknown, we expect that our molecular-dynamics simulations could inspire novel antiviral drugs based on biodegradable substances.

\section{Materials and Methods.}

A. Computational Methods. To investigate the structural and dynamical behaviour of SARS-COV2 RBD protein in water and concentrated POPC solutions, all atoms molecular dynamics (MD) simulations were conducted using the multi-GPUs version of the open-source package GROMACS $2020(58,59)$. Adopting a high-throughput approach, we ran multiple simulations in parallel on several compute nodes of the recently installed Marconi100 at CINECA, a world-class European Tier-0 system for high-performance computing (HPC).

In all these simulations, we used the OPLS-AA (60) force field together with the SPC/E water model (61). The dimensions of the simulation box were $10 \mathrm{~nm}$ in the cubic geometry, containing 30634 water molecules. The simulations with concentrated lecithin solution were performed using number of lecithin molecules $N_{L}=5, N_{L}=10, N_{L}=15$ respectively. The net charge of the protein was +2 , and therefore, two negative chloride counterions were inserted to neutralize the system. Furthermore, to assess the sensitivity of different choices of the POPC molecules positions at $t=0$ in the simulation box, three different initial conditions were generated for each concentration of the lecithin solution. A cut-off radius of $1.2 \mathrm{~nm}$ was used to create a non-bonded pair list. For the short-range non-bonded interactions a cut-off length at $1.1 \mathrm{~nm}$ was chosen for a shifted Lennard-Jones potential while the long-range electrostatic interactions were taken into account via Particle Mesh Ewald-Switch(62) (PME-switch) method with a Coulomb switching cut-off at $1.2 \mathrm{~nm}$. A long-range dispersion correction was applied for truncating the van-der-Waals interactions. All bonds were constrained using the LINCS algorithm(63). A time step of 2 fs was used for the Verlet integrator. All simulations were conducted in the canonical ensemble (NVT) at 298.5 K using the velocity-rescale thermostat(64) with a time-constant of $0.1 \mathrm{ps}$. The production runs for all simulations after initial equilibration was extended up to $1.2 \mu \mathrm{s}$. In order to assess the role of increasing concentration of lecithin, we used four different values of lecithin concentration, corresponding to $N_{\mathrm{L}}=0$ (RBD + water $), 5(\mathrm{RBD}+$ lecithin + water $), 10$ and 15 lecithin molecules in the simulation box. For each simulation containing lecithin, we ran three independent simulations each with different initial condition

B. RBM-Lecithin binding energy calculations. The binding energy of the SARS-CoV-2 receptor binding domain (RBD) and lecithin complex was calculated using the molecular mechanics Poisson-Boltzmann Surface Area (MM-PBSA) method as implemented in Gromacs. For details of the algorithm, the reader is referred to the original work (45). In this method the binding energy includes an enthalpic contribution coming from the sum of van-der-Waals and electrostatic interactions between the lecithin and protein. The contribution of the polar solvation energy to the free energy was determined by solving Poisson-Boltzmann (PB) equation, while the nonpolar contribution of the solvent effect was determined using the solvent accessible surface area (SASA) and effective surface tension.

The linear Poisson-Boltzmann equation was used to determine the polar solvation energy contribution. In these calculations, we use a dielectric constant of 80 for the solvent, and a value of 4 for that of the solute. We used two different salt concentrations of $0 \mathrm{M}$ and $0.15 \mathrm{M}$ within the Poisson-Boltzmann theory to get some theoretical error bars on how the ionic concentration affects the polar solvation energy. For the nonpolar solvation energy, a surface tension constant was set to $0.022 \mathrm{~kJ} / \mathrm{mol}\left(\AA^{2}\right)$ with a solvent probe radius of $1.4 \AA$.

\section{ACKNOWLEDGMENTS}

We thank the HPC agreement between the ICTP and CINECA for making available the computer power needed for this research. We thank Mohammad Reza Ejtehadi for valuable feedback and comments.

\section{References}

1. LC McDonald, et al., Sars in healthcare facilities, toronto and taiwan. Emerg. Infect. Dis. 10, 777-781 (2004).

2. SY Cho, et al., Mers-cov outbreak following a single patient exposure in an emergency room in south korea: an epidemiological outbreak study. Lancet 388, 994-1001 (2016).

3. LA Reperant, AD Osterhaus, Aids, avian flu, sars, mers, ebola, zika... what next? Vaccine 35, 4470-4474 (2017).

4. World health organization. coronavirus disease 2019 (covid-19)-situation report-146 (2020).

5. N Wang, J Shang, S Jiang, L Du, Subunit vaccines against emerging pathogenic human coronaviruses. Front. Microbiol. 11, 298-1-298-19 (2020).

6. J Cohen, Vaccine designers take first shots at covid-19. Science 368, 14-16 (2020).

7. TM Abd El-Aziz, JD Stockand, Recent progress and challenges in drug development against covid-19 coronavirus (sars-cov-2)-an update on the status. Infect. Genet. Evol. 83, 1043271-104327-10 (2020).

8. L Peeples, News feature: Avoiding pitfalls in the pursuit of a covid-19 vaccine. Proc. Natt. Acad. Sci. U.S.A. 117, 8218-8221 (2020). 
9. N Vankadari, Arbidol: A potential antiviral drug for the treatment of sars-cov-2 by blocking the trimerization of viral spike glycoprotein? nt. J. Antimicrob. Agents 56, 105998-1-105998-3 (2020).

10. $\mathrm{P}$ Zhou, et al., A pneumonia outbreak associated with a new coronavirus of probable bat origin. Nature $579,270-273(2020)$.

11. D Wrapp, et al., Cryo-em structure of the 2019-ncov spike in the prefusion conformation. Science 367, 1260-1263 (2020).

12. AC Walls, et al., Structure, function, and antigenicity of the sars-cov-2 spike glycoprotein. Cell 181, 281-292 (2020)

13. Y Wan, J Shang, R Graham, RS Baric, F Li, Receptor recognition by the novel coronavirus from wuhan: an analysis based on decade-long structural studies of sars coronavirus. J. Virol. 94, 1-9 (2020)

14. R Yan, et al., Structural basis for the recognition of sars-cov-2 by full-length human ace 2 . Science 367, 1444-1448 (2020).

15. A Choudhury, $\mathrm{S}$ Mukherjee, In silico studies on the comparative characterization of the interactions of sars-cov-2 spike glycoprotein with ace-2 receptor homologs and human tlrs. J. Med. Virol. 92, 2105-2113 (2020).

16. Y Wang, M Liu, J Gao, Enhanced receptor binding of sars-cov-2 through networks of hydrogen-bonding and hydrophobic interactions. Proc. Natl. Acad. Sci. U.S.A 117, 13967-13974 (2020)

17. M Amin, MK Sorour, A Kasry, Comparing the binding interactions in the receptor binding domains of sars-cov-2 and sars-cov. J. Phys. Chem. Lett. 11, 4897-4900 (2020).

18. V Monteil, et al., Inhibition of sars-cov-2 infections in engineered human tissues using clinicalgrade soluble human ace2. Cell 181, 905-913 (2020).

19. H Zhang, JM Penninger, Y Li, N Zhong, AS Slutsky, Angiotensin-converting enzyme 2 (ace2) as a sars-cov-2 receptor: molecular mechanisms and potential therapeutic target. Intensive Care Med. 46, 586-590 (2020).

20. Y Han, P Král, Computational design of ace2-based peptide inhibitors of sars-cov-2. ACS nano 14, 5143-5147 (2020).

21. B Nami, A Ghanaeian, K Ghanaeian, N Nami, The effect of ace2 inhibitor mln- 4760 on the interaction of sars-cov-2 spike protein with human ace2: a molecular dynamics study. (2020) arXiv: 10.26434/chemrxiv.12159945 (22 April 2020).

22. $\mathrm{W} \mathrm{Li}$, et al., High potency of a bivalent human vh domain in sars-cov- 2 animal models. Cell 183, 1-13 (2020).

23. L Hanke, et al., An alpaca nanobody neutralizes sars-cov-2 by blocking receptor interaction. Nat. Commun. 11, 4420-1-4420-9 (2020).

24. L Cao, et al., De novo design of picomolar sars-cov-2 miniprotein inhibitors. Science (2020) doi: 10.1126/science.abd990 (09 September 2020).

25. D Shin, et al., Papain-like protease regulates sars-cov-2 viral spread and innate immunity. Nature (2020) doi: doi:10.1038/s41586-020-2601-5 (29 July 2020).

26. B Turoňová, et al., In situ structural analysis of sars-cov-2 spike reveals flexibility mediated by three hinges. Science (2020) doi: doi:10.1126/science.abd5223 (18 August 2020).

27. A Spinello, A Saltalamacchia, A Magistrato, Is the rigidity of sars-cov-2 spike receptor-binding motif the hallmark for its enhanced infectivity? insights from all-atoms simulations. J. Phys. Chem. Lett. 11, 4785-4790 (2020).

28. R Vivek-Ananth, A Rana, N Rajan, HS Biswal, A Samal, In silico identification of potential natural product inhibitors of human proteases key to sars-cov-2 infection. Molecules 25, 3822-1-3822-28 (2020).

29. R Fleming, et al., Phospholipids for the treatment of infection by togaviruses, herpes viruses and coronaviruses (2005) US Patent App. 10/783,927.

30. M Numata, HW Chu, A Dakhama, DR Voelker, Pulmonary surfactant phosphatidylglycerol inhibits respiratory syncytial virus-induced inflammation and infection. Proc. Natl. Acad. Sci. U.S.A. 107, 320-325 (2010).

31. M Numata, et al., Phosphatidylglycerol suppresses influenza a virus infection. Am. J. Respir. Cell Mol. Biol. 46, 479-487 (2012).

32. RE Ostlund Jr, CA Spilburg, WF Stenson, Sitostanol administered in lecithin micelles potently reduces cholesterol absorption in humans. Am. J. Clin. Nutr. 70, 826-831 (1999).

33. E Nyankson, MJ DeCuir, RB Gupta, Soybean lecithin as a dispersant for crude oil spills. ACS Sustain. Chem. Eng. 3, 920-931 (2015).

34. R Shi, et al., A human neutralizing antibody targets the receptor binding site of sars-cov-2. Nature 584, 120-124 (2020).

35. S Xia, et al., Inhibition of sars-cov-2 (previously 2019-ncov) infection by a highly potent pancoronavirus fusion inhibitor targeting its spike protein that harbors a high capacity to mediate membrane fusion. Cell Res. 30, 343-355 (2020).

36. J Sun, Y Li, P Liu, J Lin, Study of the mechanism of protonated histidine-induced conformational changes in the zika virus dimeric envelope protein using accelerated molecular dynamic simulations. J. Mol. Graph. Model. 74, 203-214 (2017).

37. G Meher, S Sinha, GP Pattnaik, S Ghosh Dastidar, H Chakraborty, Cholesterol modulates membrane properties and the interaction of gp 41 fusion peptide to promote membrane fusion. J. Phys. Chem. B 123, 7113-7122 (2019).

38. U Mirastschijski, R Dembinski, K Maedler, Lung surfactant for pulmonary barrier restoration in patients with covid-19 pneumonia. Front. Med. 7, 254-1-254-4 (2020).

39. J Lan, et al., Structure of the sars-cov-2 spike receptor-binding domain bound to the ace2 receptor. Nature 581, 1-6 (2020).

40. D Otzen, Protein-surfactant interactions: a tale of many states. Biochim. Biophys. Acta 1814 562-591 (2011)

41. DM Small, M Bourgès, D Dervichian, Ternary and quaternary aqueous systems containing bile salt, lecithin and cholesterol. Nature 211, 816-818 (1966).

42. M Abrams, Isolation and quantitative estimation of pulmonary surface-active lipoprotein. J. Appl. Physiol. 21, 718-720 (1966).

43. D Tierney, $\mathrm{J}$ Clements, $\mathrm{H}$ Trahan, Rates of replacement of lecithins and alveolar instability in rat lungs. Am. J. Physiol. 213, 671-676 (1967).

44. P van Hoogevest, Review-an update on the use of oral phospholipid excipients. Eur. J. Pharm. Sci. 108, 1-12 (2017).
45. R Kumari, R Kumar, OSDD Consortium, A Lynn, g_mmpbsa a gromacs tool for highthroughput mm-pbsa calculations. J. Chem. Inf. Model. 54, 1951-1962 (2014).

46. E Wang, et al., End-point binding free energy calculation with $\mathrm{mm} / \mathrm{pbsa}$ and $\mathrm{mm} / \mathrm{gbsa}$ : strategies and applications in drug design. Chem. Rev. 119, 9478-9508 (2019).

47. C Wang, D Greene, L Xiao, R Qi, R Luo, Recent developments and applications of the mmpbsa method. Front. Mol. Biosci. 4, 87-1-87-18 (2018).

48. S Genheden, U Ryde, The $\mathrm{mm} / \mathrm{pbsa}$ and $\mathrm{mm} / \mathrm{gbsa}$ methods to estimate ligand-binding affinities. Expert. Opin. Drug Discov. 10, 449-461 (2015).

49. VK Bhardwaj, et al., Identification of bioactive molecules from tea plant as sars-cov-2 main protease inhibitors. J. Biomol. Struct. Dyn., 1-13 (2020) doi: 10.1080/07391102.2020. 1766572 (13 May 2020)

50. M Aldeghi, A Heifetz, MJ Bodkin, S Knapp, PC Biggin, Accurate calculation of the absolute free energy of binding for drug molecules. Chem. Sci. 7, 207-218 (2016).

51. PK Panda, et al., Structure-based drug designing and immunoinformatics approach for sarscov-2. Sci. Adv. 6, eabb8097-1-eabb8097-14 (2020).

52. R Vivek-Ananth, A Rana, N Rajan, HS Biswal, A Samal, In silico identification of potentia natural product inhibitors of human proteases key to sars-cov-2 infection. Molecules 25 3822-1-3822-28 (2020).

53. MC Bellissent-Funel, et al., Water determines the structure and dynamics of proteins. Chem Rev. 116, 7673-7697 (2016).

54. BJ Berne, JD Weeks, R Zhou, Dewetting and hydrophobic interaction in physical and biological systems. Annu. Rev. Phys. Chem. 60, 85-103 (2009).

55. D Chandler, Hydrophobicity: Two faces of water. Nature 417, 491-491 (2002).

56. J Lan, et al., Structure of the sars-cov-2 spike receptor-binding domain bound to the ace2 receptor. Nature 581, 215-220 (2020).

57. G Serrano, et al., Liposomal lactoferrin as potential preventative and cure for covid-19. Int. J. Res. Heal. Sci 8, 8-15 (2020).

58. MJ Abraham, et al., Gromacs: High performance molecular simulations through multi-level parallelism from laptops to supercomputers. SoftwareX 1, 19-25 (2015).

59. A Lindahl, M Hess, D van der Spoel, Gromacs 2020 source code (2020).

60. WL Jorgensen, DS Maxwell, J Tirado-Rives, Development and testing of the opls all-atom force field on conformational energetics and properties of organic liquids. J. Am. Chem. Soc. 118, 11225-11236 (1996).

61. H Berendsen, J Grigera, T Straatsma, The missing term in effective pair potentials. J. Phys. Chem. 91, 6269-6271 (1987).

62. T Darden, D York, L Pedersen, Particle mesh ewald: An $n \log (n)$ method for ewald sums in large systems. J. Chem. Phys. 98, 10089-10092 (1993).

63. B Hess, H Bekker, HJ Berendsen, JG Fraaije, Lincs: a linear constraint solver for molecular simulations. J. Comput. Chem. 18, 1463-1472 (1997).

64. G Bussi, D Donadio, M Parrinello, Canonical sampling through velocity rescaling. J. Chem Phys. 126, 014101-1-014101-7 (2007) 